\title{
DC/DC Converters Based on Hybrid MMC for HVDC Grid Interconnection
}

\author{
Rong Zeng*, Lie Xu*, and Liangzhong Yao ${ }^{\dagger}$ \\ *Department of Electronic and Electrical Engineering, University of Strathclyde, Glasgow, G1 1XW, UK \\ (emails: rong.zeng@strath.ac.uk,lie.xu@strath.ac.uk) \\ ${ }^{+}$China Electric Power Research Institute, Xiaoying Road, Beijing, 100192, China \\ (email: yaoliangzhong@epri.sgcc.com.cn)
}

Keywords: DC/DC converter, modular multilevel converter, dc fault blocking, HVDC interconnection, average modelling.

\begin{abstract}
This paper presents a multi-terminal high-power DC/DC converter configuration based on hybrid MMC topology with fault blocking capability for interconnecting HVDC systems. Its main functions include bidirectional power flow, step-up and step-down operation and fault isolation equivalent to a DC circuit breaker. By contrast to the conventional MMC based DC/DC converter, the proposed DC/DC converter with hybrid $\mathrm{MMC}$ configuration has the advantage of being able to block the DC/DC converter terminal connecting to faulty DC grid section, while continue operating the other terminals connected to healthy DC grid sections. The proposed DC/DC converter operation is analyzed and its control is described. Simulation results using Matlab/Simulink are presented to demonstrate the robust performance during $\mathrm{dc}$ fault conditions.
\end{abstract}

\section{Introduction}

HVDC transmission has become the solution for large-scale integration of renewable energy, especially for offshore wind farms over long distance $[1,2]$. Of the many technique challenges, the interconnection of DC grids with similar or different voltage levels and the power flow control among them have become one of the main barriers for the development of large scale HVDC transmission system. To solve these issues, the DC/DC converter, as a key component for the interconnection of DC grids, should have the following features: 1) bidirectional power flow; 2) step-up and step-down voltage levels; 3) DC fault blocking [3].

Due to high-voltage and high-power requirements for the DC/DC converter in HVDC system, the conventional DC/DC topologies designed for lower or medium voltage and power ranges are not suitable for HVDC applications. Modular Multilevel Converter (MMC) has become the optimal solution for HVDC applications due to its advantages of modular design, high efficiency and scalability and excellent output waveform with low harmonic distortion, etc. [4, 5]. There are several papers referring to using two MMC configuration connected by a medium/high-frequency transformer to compose a DC/DC converter [3, 6-10]. In [8], a DC/DC converter formed by two MMCs in a back-to-back configuration including a transformer was proposed. In case of a short circuit on one side, both primary and secondary converters are blocked and the short circuit current through the converters can efficiently be avoided, so as to remedy the critical issue of the conventional MMC topology on DC fault protection in multi-terminal dc grids. Although such DC/DC configuration can handle DC fault without additional circuits, it has to block both the primary and secondary side MMCs simultaneously to isolate the fault. For a DC/DC converter with more than two terminals connecting large number of DC grid sections, DC fault on one section will lead to the blocking of all the converters in the DC/DC converter which will affect the power transmission among the healthy DC sections in such a multi-section HVDC interconnection system.

To tackle the above mentioned issues, in this paper, an improved 3-terminal DC/DC converter based on a simplified hybrid MMC configuration is proposed for multi-terminal DC grids interconnection. In contrast to the basic hybrid MMC with half half-bridge SM (HBSM) and half full-bridge SM (FBSM) in each arm [11, 12], this MMC structure is a simplified configuration with less semiconductor devices, but also has the dc fault blocking capability. With such advantages, the proposed DC/DC converter can isolate the faulty DC grid section, while continue operating the healthy DC grid sections.

The paper is organized as follows. Section 2 outlines the proposed three-terminal DC/DC converter interconnecting three DC grid sections. The simplified hybrid MMC based DC/DC topology and its corresponding control strategy under normal operation conditions and dc fault conditions are proposed in Section 3. Average converter model for the hybrid MMC is described in Section 4 and case studies are carried out and corresponding simulation results are presented in Section 5 to demonstrate the power flow control and DC fault management of the proposed DC/DC converter. Finally, Section 6 draws the conclusions. 


\section{System configuration}

Fig.1 presents the proposed three-terminal DC/DC converter interconnecting three independent HVDC grid sections. It is consisted of a DC grid section connecting to an offshore wind farm, DC Grid 2 and DC Grid 3 with similar or different voltage levels. The 3-terminal DC/DC converter operates as a $\mathrm{DC}$ hub in the system to connect and manage power flow among these three DC grids. It comprises three simplified hybrid MMC with two isolation transformers, as shown in Fig. 2(a). In order to reduce the footprint of the transforms, the ac output of the hybrid MMC can be higher than nominal AC system frequencies of $50 / 60 \mathrm{~Hz}$, e.g., hundreds $\mathrm{Hz}$.

In contrast to the basic hybrid MMC with half half-bridge and half full-bridge SMs in each arm [11, 12], this MMC structure is a simplified configuration by removing an IGBT in the fullbridge SMs (as shown as $\mathrm{S}_{3}$ in Fig. 2(b)). Compared to the conventional HBSM, it requires one extra switch and two diodes for a hybrid SM. The fault current path in each SM during a dc fault is also indicated in Fig. 2(c). It is known that if the total voltage from the series capacitors in the FBSMs within the fault current path is higher than the ac voltage, the dc fault can be blocked. According to the analysis in [11], the MMC configuration with half HBSMs and half FBSMs can provide enough capacitor voltage to oppose the ac voltage, and therefore the simplified hybrid MMC inherently has the dc fault blocking capability. With the advantage of inherent dc fault blocking capability, in case of dc fault happened in one DC grid (i.e. DC Grid 3 in Fig. 1), the Hybrid MMC3 can be blocked and the other two (Hybrid MMC1 and Hybrid MMC2 shown in Fig. 1) can continue operating and transmitting power between Grid 1 and Grid 2.

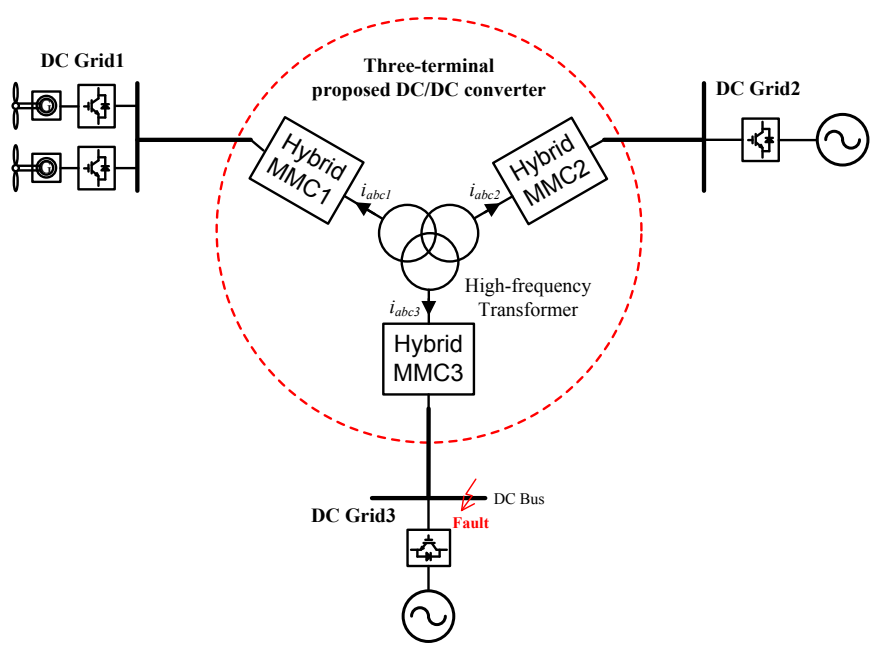

Fig. 1 Layout of a three interconnected DC grids

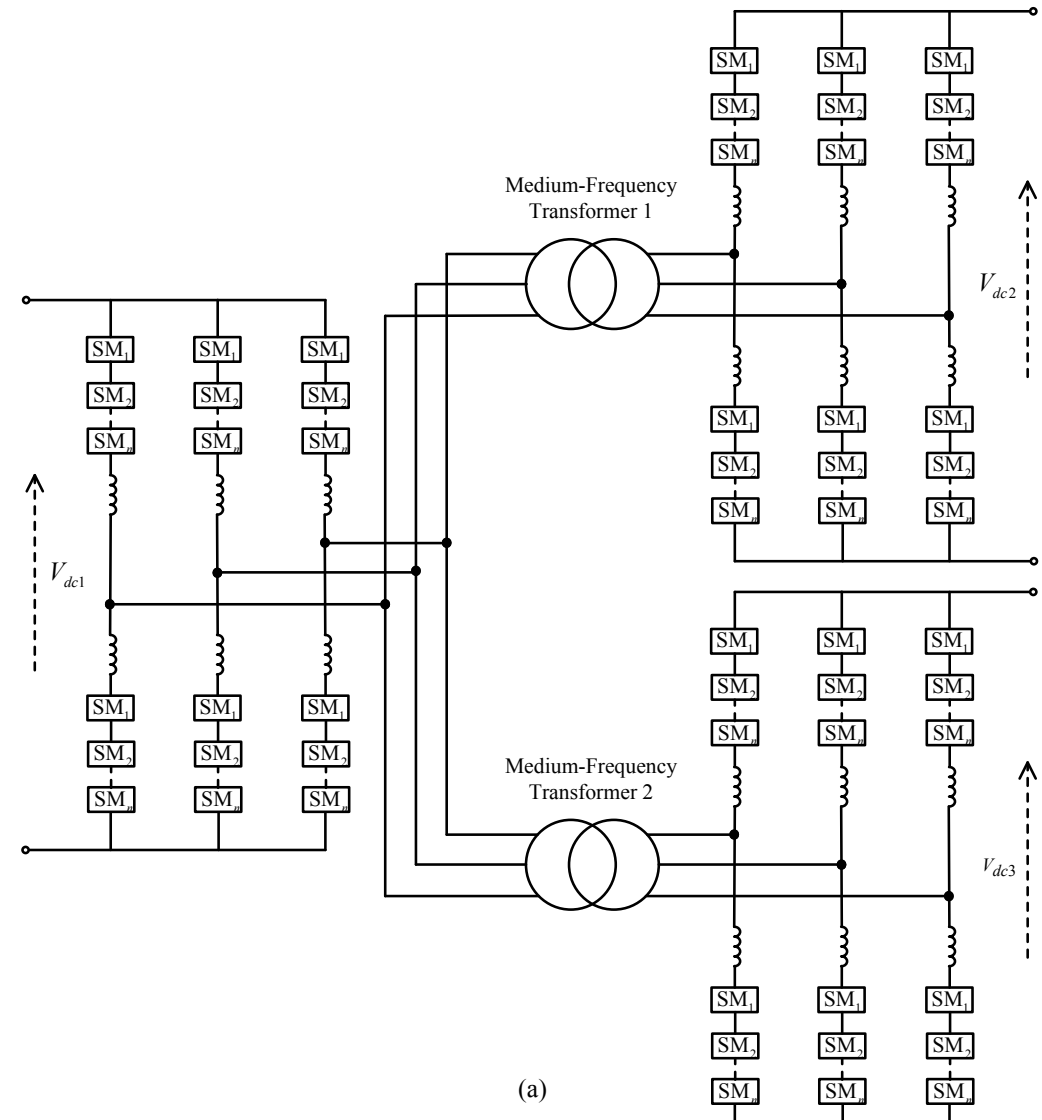

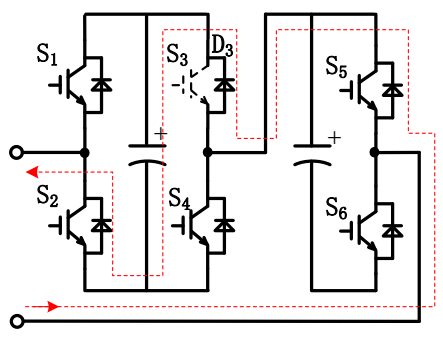

(b)

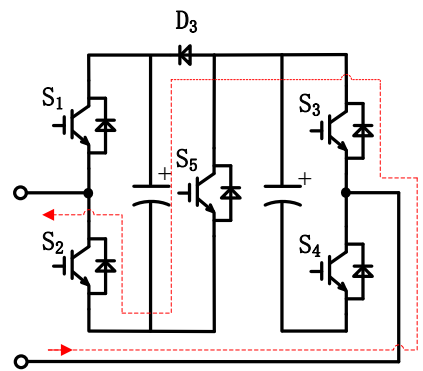

(c)

Fig. 2 Configuration of the proposed hybrid MMC based DC/DC converter 


\section{Control strategy for the proposed DC/DC converter}

The DC/DC converter is consisted of a number of MMC based $\mathrm{DC} / \mathrm{AC}$ converters depending on the number of $\mathrm{DC}$ terminal required. Among the different MMC converters, one MMC operates as a voltage source to generate mediumfrequency ac voltage for the common $\mathrm{AC}$ side and the other converters control their power exchanges between the $\mathrm{AC}$ and DC side so as to control the power flow among the connected different DC sections.

The detailed structure of one MMC leg is given in Fig. 3, where $V_{d c}$ is the dc-link voltage and $L$ is the arm inductor in each arm. $v_{p a}$ and $v_{n a}$ are the total voltages generated by all the SMs in the upper and lower arms, respectively. $i_{p a}$ and $i_{n a}$ are the current of the upper and lower arms, respectively. $i_{a}$ is the output ac phase current.

According to Fig. 3, the arm current $i_{p a}$ and $i_{n a}$ can be expressed as $[4,5]$

$$
\begin{gathered}
i_{p a}=i_{a} / 2+i_{c c a} \\
i_{n a}=-i_{a} / 2+i_{c c a}
\end{gathered}
$$

where $i_{c c a}$ is the common mode current in phase $a$ flowing through both the upper and lower arms. It has no effect on the output ac phase current and can be expressed as:

$$
i_{c c a}=\left(i_{p a}+i_{n a}\right) / 2
$$

Taking the neutral point $n$ of the dc link as the voltage reference, the voltage and current can be expressed as

$$
L_{0} \frac{d i_{p a}}{d t}=e_{a}-v_{a n}, \quad L_{0} \frac{d i_{n a}}{d t}=v_{a n}-e_{a}
$$

where $e_{a}$ is the equivalent phase voltage in phase $a$ and is expressed as

$$
e_{a}=V_{d c} / 2-v_{p a}=-V_{d c} / 2+v_{n a}=v_{n a}-v_{p a} / 2
$$

For the operation of a MMC, the following equation defines the dc voltage and must be satisfied under any conditions

$$
v_{p a}+v_{n a}=V_{d c}
$$

Combine (5) and (6), the required arm voltage references are calculated as

$$
v_{p a_{-} r e f}=V_{d c} / 2-e_{a}, \quad v_{n a_{-} r e f}=V_{d c} / 2+e_{a}
$$

One of the primary requirements for the MMC inverter operating as a voltage source is to generate a mediumfrequency ac voltage with constant amplitude and frequency. The schematic diagram of the control strategy for the MMC operating as a voltage source is shown in Fig. 4. It is consisted of double control loops, including an outer voltage loop with proportional-integral (PI) controllers to control the $d$ - and $q$ - axis voltage space vector, and an inner current loop with PI controllers to track the ac current reference. The $d$ axis voltage reference $V_{i d}^{*}$ is set to be rated ac phase voltage peak value and $V_{i q}^{*}$ is set to be 0 . The ac voltage frequency is set to a constant of $f_{i}$. The output of the control scheme is the equivalent phase voltage $e$. substituting into (7), the required arm voltage references can be calculated. Since the capacitor voltage balancing algorithm and PWM modulation method have been well documented $[4,5]$, no further details are given here.

For the other MMCs, the task is to control the power flows between the local medium-frequency ac voltage and connected DC terminals. The schematic diagram of the control strategy for the MMC rectifier is shown in Fig. 5 where reactive power reference (q-axis current) can be simply set to zero. It also includes double control loops, an outer voltage loop with proportional-integral (PI) controllers to control the active and reactive power tracking the reference value, and an inner current loop with PI controllers to track the ac current reference.

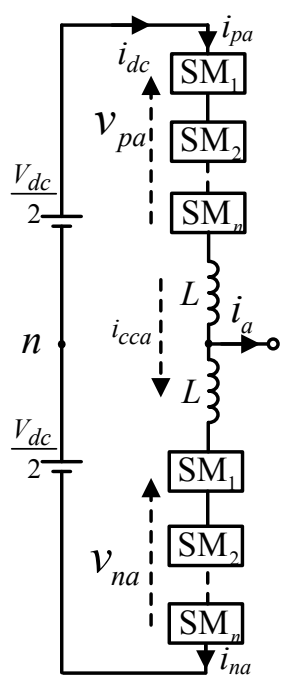

Fig.3 Basic structure of one leg in MMC

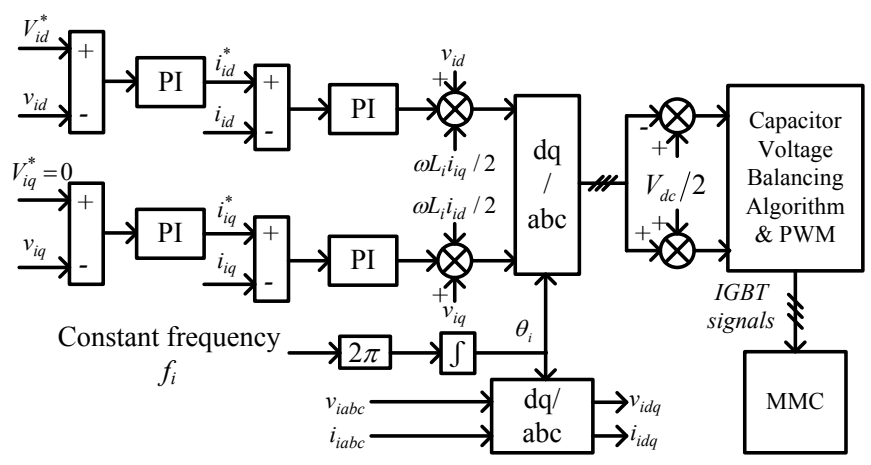

Fig. 4 Schematic diagram of the controller for the MMC inverter 


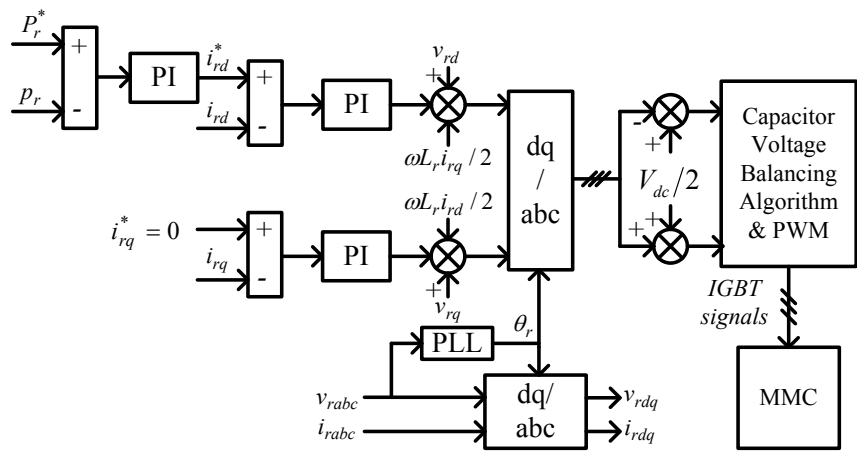

Fig. 5 Schematic diagram of the controller for the MMC rectifier

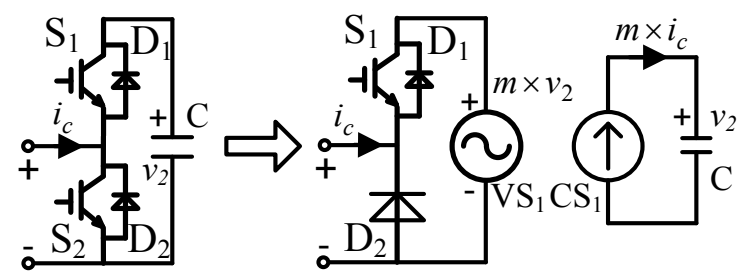

(a) Average modelling for the HBSM

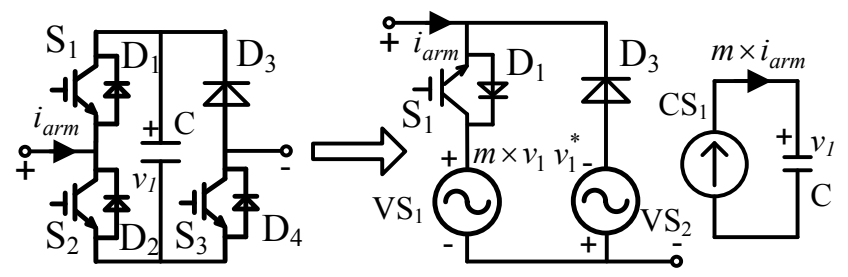

(b) Average modelling for the FBSM

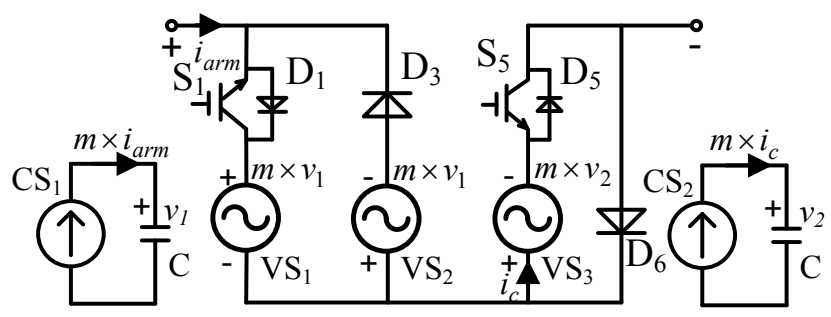

(c) Average modelling for the hybrid SM

Fig. 6 Average modelling replacing SMs in each arm of the hybrid MMC

\section{Converter average model development}

It is known that detailed modelling of MMCs consisted of huge number of switching devices, can result in excessive computational complexity and long simulation time. For a multi-terminal interconnected DC grids system, it is not efficient and practicable to using detailed models to build the whole system [13, 14]. In order to solve this issue, a simplified simulation model using average converter model for the proposed DC/DC converter using hybrid MMC topology is adopted. The average model replaces the strings of cells in each arm with a single average value cell as shown in Fig. 6. It is consisted of coupled controllable voltage source and controlled current source. Since the basic building block in the hybrid MMC is one simplified FBSM and one HBSM, all capacitors in one arm are lumped together and modelled as a single capacitor C. Fig. 6(a) and Fig. 6(b) show the average modelling for the HBSM and the FBSM, respectively. The capacitor is replaced by a controllable voltage source which is fed by a controllable current source. The reference value for the controllable current source is the product of input current and modulation index $m$. The voltage built up across the arm is given by the number of cells that being switched on, and this is modelled as a controllable voltage source whose reference value is the product of the capacitor voltage and the modulation waveform.

Additional semiconductor devices have been added so that the model can accurately replicate all possible current paths in a real converter during DC faults. As shown in the right hand side of Fig. 6(a), during normal operation in the HBSM, switch $S_{1}$ is closed, allowing current to flow in both the positive and negative directions through $S_{1}$ or $D_{1} \cdot D_{2}$ is reverse biased. For the FBSM average model shown in on the right hand side of Fig. 6(b), during normal operation, switch $\mathrm{S}_{1}$ is closed and $\mathrm{D}_{3}$ is reverse biased. The modulation index is derived from the arm reference voltage derived from (7). Under the dc fault conditions, all IGBTs should be blocked in the detailed model, which is replicated by switching off $S_{1}$ in the average model and the diode $\mathrm{D}_{3}$ offers the fault current path. For the FBSM, when switch $\mathrm{S}_{1}$ switches off, the controllable voltage source $\mathrm{V}_{\mathrm{S} 2}$ (equals $v_{l}$ ) would replicate the blocking voltage formed by the FBSM capacitor voltages.

Fig. 6(c) presents the average modelling for the hybrid SM. In case of a dc fault, all IGBTs are blocked, the fault current bypasses the controllable voltage source $\mathrm{V}_{\mathrm{S} 3}$ (capacitors in the HBSMs) through $\mathrm{D}_{6}$ but flows through the controllable voltage source $V_{S 2}$ and $D_{3}$, which matches the characteristics of the actual hybrid SM shown in Fig. 2(c).

\begin{tabular}{|c|c|c|}
\multicolumn{2}{|c|}{ TABLE I PARAMETERS OF THE SIMULATION HYBRID HVDC SYSTEM } \\
\hline \multirow{2}{*}{$\begin{array}{c}\text { Hybrid } \\
\text { MMC1 }\end{array}$} & Items & Values \\
\cline { 2 - 3 } & Rated power & $1000 \mathrm{MW}$ \\
\cline { 2 - 3 } & AC side voltage & $400 \mathrm{kV}, 500 \mathrm{~Hz}$ \\
\cline { 2 - 3 } & DC side voltage & $800 \mathrm{kV}$ \\
\cline { 2 - 3 } & Number of SMs & 320 \\
\hline Hybrid & Equivalent capacitance per SM & $2.1 \mathrm{uF}$ \\
\cline { 2 - 3 } MMC2 & Rated power & $300 \mathrm{MW}$ \\
\cline { 2 - 3 } & AC side voltage & $600 \mathrm{kV}$ \\
\cline { 2 - 3 } & DC side voltage & 240 \\
\cline { 2 - 3 } & Number of SMs & $1.8 \mathrm{uF}$ \\
\cline { 2 - 3 } & Equivalent Capacitance per SM & $500 \mathrm{kV} / 300 \mathrm{kV}$ \\
\hline Hybrid & Transformer 1 & $500 \mathrm{MW}$ \\
\cline { 2 - 3 } MMC3 & Rated power & $400 \mathrm{kV}, 500 \mathrm{~Hz}$ \\
\cline { 2 - 3 } & AC side voltage & $400 \mathrm{kV}$ \\
\cline { 2 - 3 } & DC side voltage & 160 \\
\cline { 2 - 3 } & Number of SMs & $4.2 \mathrm{uF}$ \\
\cline { 2 - 3 } & Equivalent Capacitance per SM & \\
\cline { 2 - 3 } & Transformer 2 & $\mathrm{kV}$ \\
\hline
\end{tabular}

\section{Case studies}

To verify the above mentioned configuration and control strategy of the proposed DC/DC converter, a three-terminal DC/DC converter model shown in Fig. 1 is developed using Matlab/Simulink. MMC1 operates as a voltage source to generate an $\mathrm{AC}$ voltage with constant amplitude of $400 \mathrm{kV}$ 
(phase-phase rms.) and a constant frequency of $500 \mathrm{~Hz}$. MMC2 and MMC3 control their active power flow to DC Grid 1 and DC Grid 2. The main parameters are listed in Table I. Due to the use of higher frequency, the cell capacitor size can be significantly reduced compared to normal MMC operating at line frequency.

Fig. 7 shows the steady-state operation of the proposed DC/DC converter. The frequency of ac voltage generated by MMC1 is $500 \mathrm{~Hz}$, and the power flowing direction is from $\mathrm{MMC} 1$ to $\mathrm{MMC} 2$ and $\mathrm{MMC} 3$. The consuming active power at MMC2 and MMC3 is equal (0.5 p.u.), and the reactive power is 0 . It can be observed that the ac voltage generated by MMC1 is sinusoidal with constant amplitude and constant frequency, and MMC2 and MMC3 can precisely track the reference value of active and reactive power.
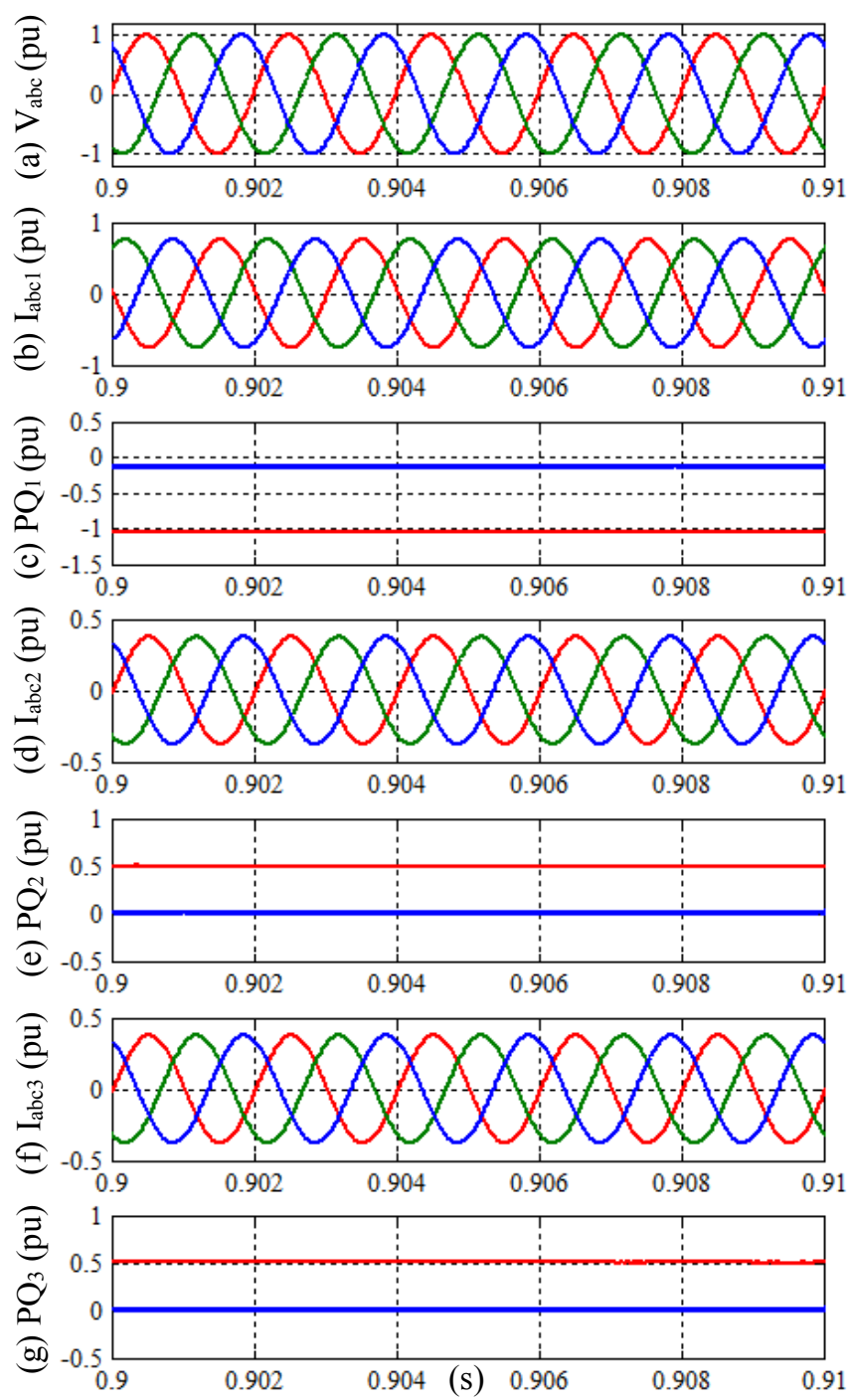

Fig. 7 Simulation results demonstrating the steady-state operation performance of the proposed $\mathrm{DC} / \mathrm{DC}$ converter, (a) ac voltage at the ac transformer; (b) ac current at $\mathrm{MMC1}$; (c) active and reactive power at MMC1; (d) ac current at MMC2; (e) active and reactive power at MMC2; (f) ac current at MMC3; (g) active and reactive power at MMC3.
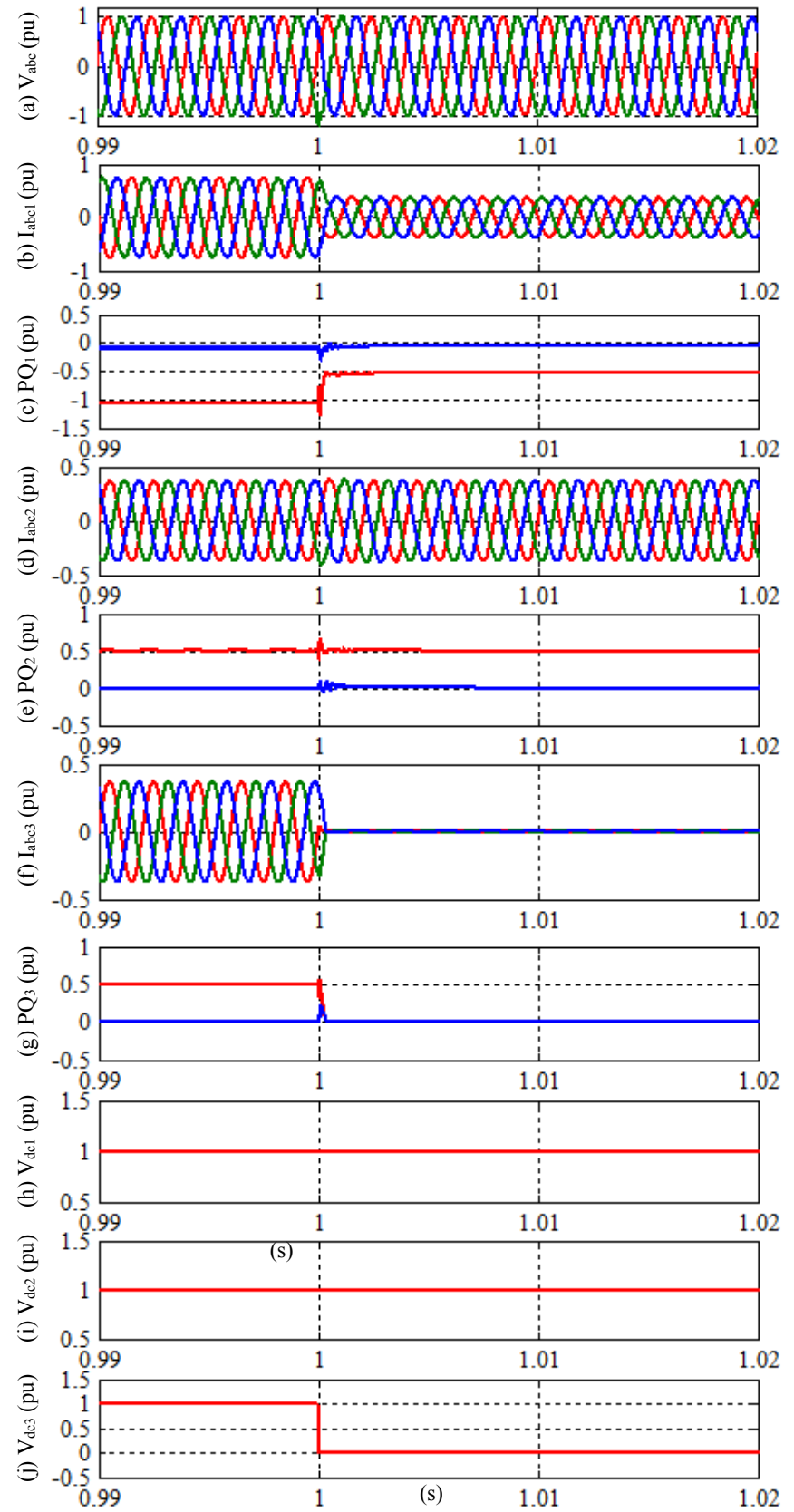

Fig. 8 Simulation results demonstrating the behaviours of the proposed DC/DC converter under dc fault conditions, (a) ac voltage at the ac transformer; (b) ac current at $\mathrm{MMC1}$; (c) active and reactive power at MMC1; (d) ac current at MMC2; (e) active and reactive power at MMC2; (f) ac current at MMC3; (g) active and reactive power at MMC3; (h) dc voltage at $\mathrm{MMC} 1$; (i) de voltage at MMC2; (j) dc voltage at MMC3.

Fig. 8 shows the behaviours of the system under dc fault conditions. When a de fault occurs at MMC3 side $(t=1.0 \mathrm{~s})$, there are large fault current and the dc voltage collapses at MMC3 side, which would affect the medium-frequency ac voltage generated by $\mathrm{MMC} 1$ in the transformer. It can be seen that the ac current and active and reactive power in MMC2 side are also influenced by the affected ac voltage. As the fault detected, the MMC3 is blocked, the fault current iabc3 goes to zero quickly and the fault is isolated. The other two 
MMCs (MMC1 and MMC2) continue operating without blocking. It is demonstrated that the proposed $\mathrm{DC} / \mathrm{DC}$ converter can quickly isolate the local dc fault and remain the other healthy parts of system operating without blocking.

\section{Conclusion}

A high power DC/DC converter based on hybrid MMC topology with fault blocking capability for interconnecting large HVDC systems has been studied in this paper. The configuration and control strategy for the proposed DC/DC converter have been presented. The proposed converter is able to continue operating the healthy parts of the system rather than blocking the complete $\mathrm{DC} / \mathrm{DC}$ converter in the conventional MMC-based DC/DC converter system. Simulation results using a 3-terminal DC/DC converter connecting three separate DC sections are presented to demonstrate the robust performance of the proposed configuration during a DC fault on one DC network section.

\section{Acknowledgements}

Mr. R. Zeng would like to thank the Chinese Scholarship Council (CSC) for partially sponsoring his Ph.D. study in University of Strathclyde, Glasgow, U.K. Financial support from China Electric Power Research Institute is also appreciated.

\section{References}

[1] N. Flourentzou, V. G. Agelidis, and G. D. Demetriades, "VSC-Based HVDC Power Transmission Systems: An Overview," IEEE Trans. Ind. Appl., vol. 24, pp. 592602, 2009.

[2] L. Xu, L. Yao, and C. Sasse, "Grid Integration of Large DFIG-Based Wind Farms Using VSC Transmission," IEEE Trans. Power Sys., vol. 22, pp. 976-984, 2007.

[3] G. J. Kish, M. Ranjram, and P. W. Lehn, "A Modular Multilevel DC/DC Converter With Fault Blocking Capability for HVDC Interconnects," Power Electronics, IEEE Transactions on, vol. 30, pp. 148-162, 2015.

[4] M. Saeedifard and R. Iravani, "Dynamic performance of a modular multilevel back-to-back HVDC system," IEEE Trans. Power Del., vol. 25, pp. 2903-2912, 2010.
[5] Q. R. Tu, Z. Xu, and L. Xu, "Reduced SwitchingFrequency Modulation and Circulating Current Suppression for Modular Multilevel Converters," IEEE Trans. Power Del., vol. 26, pp. 2009-2017, 2011.

[6] S. Kenzelmann, D. Dujic, F. Canales, Y. R. de Novaes, and A. Rufer, "Modular DC/DC converter: Comparison of modulation methods," in Power Electronics and Motion Control Conference (EPE/PEMC), 2012 15th International, 2012, pp. LS2a.1-1-LS2a.1-7.

[7] S. Kenzelmann, A. Rufer, D. Dujic, F. Canales, and Y. R. de Novaes, "Isolated DC/DC Structure Based on Modular Multilevel Converter," Power Electronics, IEEE Transactions on, vol. 30, pp. 89-98, 2015.

[8] S. Kenzelmann, A. Rufer, M. Vasiladiotis, D. Dujic, F. Canales, and Y. R. de Novaes, "A versatile DC-DC converter for energy collection and distribution using the Modular Multilevel Converter," in Power Electronics and Applications (EPE 2011), Proceedings of the 201114th European Conference on, 2011, pp. 1-10.

[9] T. Luth, M. M. C. Merlin, T. C. Green, F. Hassan, and C. D. Barker, "High-Frequency Operation of a DC/AC/DC System for HVDC Applications," Power Electronics, IEEE Transactions on, vol. 29, pp. 41074115, 2014.

[10] A. Schon and M. M. Bakran, "A new HVDC-DC converter with inherent fault clearing capability," in Power Electronics and Applications (EPE), 2013 15th European Conference on, 2013, pp. 1-10.

[11] R. Zeng, L. Xu, L. Z. Yao, and B. W. Williams, "Design and Operation of a Hybrid Modular Multilevel Converter," IEEE Trans. Power Electron., vol. PP, pp. $1-1,2014$.

[12] R. Zeng, L. Xu, L. Z. Yao, and J. Morrow, "Precharging and DC Fault Ride-Through of Hybrid MMC Based HVDC Systems," IEEE Trans. Power Del., vol. PP, pp. 1-1, 2014.

[13] D. C. Ludois and G. Venkataramanan, "Simplified Terminal Behavioral Model for a Modular Multilevel Converter," Power Electronics, IEEE Transactions on, vol. 29, pp. 1622-1631, 2014.

[14] X. Jianzhong, Z. Chengyong, L. Wenjing, and G. Chunyi, "Accelerated Model of Modular Multilevel Converters in PSCAD/EMTDC," Power Delivery, IEEE Transactions on, vol. 28, pp. 129-136, 2013. 